\title{
Pensions with endogenous and stochastic fertility ${ }^{1}$
}

\author{
Helmuth Cremer, Firouz Gahvari and Pierre Pestieau \\ University of Toulouse (IDEI and GREMAQ) \\ 21, allée de Brienne \\ 31000 Toulouse - France \\ Department of Economics \\ University of Illinois at Urbana-Champaign \\ Urbana, IL 61801, USA \\ CORE, Delta, CEPR and CREPP, University of Liège \\ 7, bd du Rectorat \\ 4000 Liège - Belgique
}

September 2004

Revised, March 2006

\footnotetext{
${ }^{1}$ This paper has been presented at the CESifo Public Sector Economics Area Conference (Munich, May 2004) and at the Third Workshop of the FINRET network. We would like to thank all participants and, in particular our discussants, Henning Bohn and François Maniquet for their comments. The current version has benefitted substantially from the comments and suggestions of two anonymous referees of this Journal, and the Editor, Emmanuel Saez.
} 


\begin{abstract}
This paper studies the design of a pay-as-you-go social security system in an overlapping generations model where fertility is in part stochastic and in part determined through capital investment. If investments are publicly observable, pension benefits must be linked positively to the level of investment, and payroll taxes negatively to the number of children. The outcome is characterized by full insurance with all parents, regardless of their number of children, enjoying identical consumption levels. Without observability, benefits must increase, and payroll taxes decrease, with the number of children. The second-best level of investment, and the resulting average fertility rate, are less than their corresponding first-best levels.
\end{abstract}

JEL classification: H55; J13.

Keywords: pay-as-you-go social security, endogenous fertility, storage, moral hazard. 


\section{Introduction}

The recent fertility decline in the West is often cited as a major impediment to the fiscal solvency of pay-as-you-go (PAYGO) social security systems. At the same time, the pay-as-you-go feature of the social security systems has partly been blamed for causing the observed fertility decline. The reason for this latter linkage is that in such systems, each person's fertility decision, via its impact on the economy's population growth rate, affects everybody's pension benefits. Specifically, an increase in the rate of population growth increases the number of future workers who will have to support a retired person. No individual, however, takes this impact into account leading to a decentralized equilibrium outcome with too few children. ${ }^{1}$

The above problem is exacerbated by another externality problem associated with the "quality" of children, and their human capital accumulation, through educational decisions of the parents. The rate of return of a pay-as-you-go system depends not just on the fertility rate, but also on productivity growth. The more productive the children, the higher will be their ability to produce and to pay taxes. This reinforces the public good nature of a family's child-rearing activities. It is not surprising then that some economists have recently advocated a policy of linking pension benefits (or contributions) to individuals' fertility choices. ${ }^{2}$

Such a policy raises a number of objections, however. What truly determines fertility, and what accounts for the observed evolution in fertility behavior, are still open questions. What is clear though is that no one can fully control fertility. The actual number of children in a family does not necessarily coincide with the number the parents initially intended to have. Infertility, premature death, misplanning and multiple births are some of the reasons explaining this gap. Similarly, one cannot deterministically determine the future earning abilities of children simply by investing in their education and training. Making benefits independent of the number of children can then be viewed

\footnotetext{
${ }^{1}$ This argument emphasizes the "intergenerational transfer effect," and ignores the "capital dilution effect," associated with an increase in fertility. See the discussion below in subsection 3.4 and particularly footnote 13 .

${ }^{2}$ Abio et al. (2004), Bental (1989), Cigno et al. (2003), Fenge and Meier (2004), Kolmar (1997), van Groezen et al. $(2000,2003)$.
} 
as a mechanism to insure parents against these various random shocks. This raises the question of balancing the benefits of providing insurance to the population against the costs of their not having the "correct" number of children because of the externality problem.

Three recent papers in this Journal address different aspects of these problems. van Groezen et al. (2003) study the structure of pension plans and child allowances in an overlapping generations model where fertility is endogenous but fully deterministic. In this setting, the question of insurance does not arise. Nor will there be a moral hazard (incentive) problem as long as the number of children are publicly observable. All individuals can then be enticed to choose the socially optimal first-best number of children which corrects for the externality. Cigno et al. (2003) also consider a model in which fertility is endogenous and fully deterministic. They nevertheless bring up the question of the trade-off between insurance and incentive through children's quality ("lifetime tax contributions"), which they assume to be in part random and in part determined through costly (and possibly unobservable) actions of the parents. The moral hazard problem surfaces in their model also, when the parents' actions are unobservable. In contrast to van Groezen et al., who have an explicit two-period model, Cigno et al.'s setup is static. This aspect prevents one from distinguishing between tax and transfer policies over the individuals' life cycle-An issue that lies at the heart of the design of pension plans in overlapping generations models à la Samuelson.

The third paper is Sinn (2004) who is interested more in examining the properties of a traditional PAYGO system rather than the design of an optimal pension plan. He considers a model in which fertility is fully random where the parents are either fertile (having a fixed number of children) or infertile. In this way, he introduces the idea of a PAYGO pension plan serving as an insurance mechanism against infertility. He is also concerned with the quality of children which he models deterministically. He discusses moral hazard in terms of the distortion caused by contributions to the PAYGO system which acts as a tax on the returns to investment in educating children.

The key distinguishing element between decisions on quantity and quality of children is one of timing. The number of children born is known quite early; the quality of 
children (i.e. their future earning capacity) is determined much later. To account for both features one needs a model with at least three periods of decision making. This makes the design of an optimal pension system rather complicated to study. ${ }^{3}$ We thus ignore the externality associated with education decisions and concentrate solely on fertility. This allows us to consider a setting with two periods of decision making, as opposed to three, which simplifies the modeling substantially. Specifically, we study the design of pension systems, within the Samuelson's (1958) overlapping generations framework, when the average fertility rate in the society (the "biological interest rate") is determined endogenously. Moreover, we allow for the individual fertility rates to be not only endogenous but stochastic as well. We thus postulate that a prospective parent's actual number of children is in part random and in part the result of some early investment decision the parent makes at the beginning of the first period. We further assume that the number of children is observed early and the parents can adjust both their first and the second period consumption levels accordingly.

Given the stochastic nature of fertility, we study the design of optimal pension systems under two different informational structures. In one, we assume that the prospective parents' investments (efforts) in having children are publicly observable. In the second, we assume that the parents' investment levels are private information. This introduces a moral hazard dimension into the problem. Another underlying agency problem that surfaces, when one is dealing with endogenous fertility, is adverse selection. It arises if individuals differ in child-rearing ability, or in taste for children. To simplify matters, and to distinguish between the implications of adverse selection and moral hazard, this paper focuses solely on the moral hazard issue leaving the adverse selection considerations to another paper. ${ }^{4}$ This allows us to work with (ex-ante) identical

\footnotetext{
${ }^{3}$ Cigno and Luporini (2003) have a three-period model; however they do not optimize over tax instruments.

${ }^{4}$ See, Cremer et al. (2004). The moral hazard problem has also been studied by Cremer et al. (2003). That paper was based on two very restrictive assumptions which we drop here. First, we had ignored all possibilities for private savings, assuming that the only mechanism for transfer of resources to the future is (except for possible "voluntary" arrangements between parents and children whereby children help their retired parents with the expectation that their own children would help them) a PAYGO public pension system. Second, we had assumed that the number of children is observed late in the first period so that the first-period consumption could not vary with the number of children.
} 
individuals.

If fertility were fully deterministic, as in Groezen et al. (2003), the questions of insurance and moral hazard (incentive) would not arise. All individuals can then be enticed to choose the socially optimal first-best number of children which corrects for the externality. On the other hand, if fertility were fully random and independent of the parents' investment, there would be no externality and moral hazard (incentive) problems - only insurance considerations. The optimal social security system then is one that fully insures parents against the fertility uncertainty.

When fertility is determined in part through investment and in part through random elements, there naturally arises a question as to the possible tradeoff between full insurance and incentive considerations. In a first-best environment, when the parents' investments in having and raising children are publicly observable, one may be able to achieve the two objectives of internalizing the externality and full insurance. We show that this is the case. We also show that the decentralization of the first best requires pension benefits to be linked positively to the parents' level of investment in children (and not to their number), coupled with payroll taxes that vary inversely with the number of children. The design of an optimal social security system when investments are publicly observable but individuals' fertility rates are stochastic, is one dimension along which we generalize van Groezen et al. (2003).

The more important problem that does not arise in van Groezen et al. (2003) is that of moral hazard. When the parents' investment in children is not publicly observable, a tradeoff between insurance and incentive considerations surfaces. We prove that in this case, the optimal (second-best) level of investment in children, and the resulting average fertility rate, are less than their corresponding first-best values. To attain the secondbest, one must institute a pay-as-you-go pension plan under which benefits increase, and payroll taxes decrease, with the number of children. Moreover, families with more children should be more than compensated for the extra cost of children so that they will enjoy a higher level of first-period consumption. Interestingly, with the exception of the last finding, these results carry over to situations where payroll taxes cannot depend on the number of children. 


\section{The basics}

Consider a two-period overlapping generations model in the steady state. Each generation consists of a continuum of identical individuals. The young have fixed endowments $y$ and the old live on pensions. Preferences of the young depend positively on their consumption in the first period, $c$, and their consumption in the second period, $d$. We depart from the bulk of the literature in leaving the "love for children" out of the individuals' preferences. ${ }^{5}$ This simplification has the advantage of putting the problem into a sharper focus: Parents are not interested in children but the society is (in order to have them finance pension benefits). This was an important aspect of Samuelson's (1958) original analysis; preserving it makes the comparisons of our results to his more natural.

A parent can have either $n_{1}$ or $n_{2}$ children, with $n_{2}>n_{1}$. The actual realization of $n_{i}$ depends on an initial investment in children, $k$, and on some random shock. Thus when a parent invests $k$, he will have $n_{2}$ children with probability $\pi(k)$ where $0 \leqslant \pi(k) \leqslant 1$ and $\pi^{\prime}(k)>0\left(\pi^{\prime \prime}(k)<0\right.$ and $\left.\pi(0)>0\right)$. Naturally, the probability of having $n_{1}$ children is given by $1-\pi(k)$. Whenever it makes the notation simple, we substitute $\pi_{2}(k)$ for $\pi(k)$ and $\pi_{1}(k)$ for $1-\pi(k)$. The cost of having children is not limited to the initial investment $k$. There are other costs that vary proportionately (at the rate of $\theta \geq 0$ ) to the actual number of children. These costs are also borne in the first period.

To keep the model simple, assume that preferences over $\left(c_{i}, d_{i}\right), i=1,2$, are represented by an additive utility function. Consequently, at the beginning of the first period, the expected utility of the young (i.e. future parents) is written as

$$
U=\sum_{i=1}^{2} \pi_{i}(k)\left[u\left(c_{i}\right)+v\left(d_{i}\right)\right],
$$

where $u(\cdot)$ and $v(\cdot)$ are strictly concave functions.

There are two potential mechanisms for financing second-period consumptions: stor-

\footnotetext{
${ }^{5}$ Introducing the love for children into individuals' preferences is an easy undertaking. This would generally imply that parents would opt for a higher investment level in children thus lowering the instances under which parents may decide not to invest in children. However, as long as there is no conflict between the parents' and the society's love for children, this will not add to the scope of our analysis in terms of policy design.
} 
age or a PAYGO pension plan. Under the storage technology, part of the initial endowment is invested yielding a fixed rate of return, $r .{ }^{6}$ Under a PAYGO scheme, the government collects taxes from the current young and distributes the proceeds to the retired. With the young having, on average,

$$
\bar{n}(k)=\pi_{1}(k) n_{1}+\pi_{2}(k) n_{2}
$$

children, the PAYGO rate of return is $\bar{n}(k)-1$. This corresponds to what Samuelson called the biological rate of interest.

Observe that while the number of children of a particular family is random, the fertility rate for the society is not. It is determined fully by the choice of $k$. This property, and the existence of a storage technology with a fixed rate of return, imply that it is possible for the society to transfer resources across different generations in a deterministic fashion, and at a rate which is independent of the total savings in the economy. These two properties also imply that the only consideration in choosing between the storage technology and a pension plan, for transfer of resources, is to determine which mechanism has a higher implied rate of return. In turn, this has the strong implication that one of the two mechanisms will always dominate the other. It will never be optimal to use storage and PAYGO simultaneously. This feature, which was another fundamental aspect of Samuelson's (1958) model, allows us to isolate the effects of making fertility endogenous and stochastic on the choice between the two mechanisms.

\subsection{Laissez faire}

Absent any government intervention, each individual maximizes his expected utility subject to two budget constraints, one of which becoming relevant ex post, depending on the number of children. The Lagrangian expression associated with the individual's problem is

$$
\mathcal{L}_{L}=\sum_{i}\left\{\pi_{i}(k)\left[u\left(c_{i}\right)+v\left(d_{i}\right)\right]+\lambda_{i}\left[y-c_{i}-\frac{d_{i}}{1+r}-k-n_{i} \theta\right]\right\} .
$$

\footnotetext{
${ }^{6}$ The rate of return is net of any "capital depreciation".
} 
It follows from the first-order conditions of this problem with respect to $c_{i}$ and $d_{i}$ that

$$
\frac{v^{\prime}\left(d_{i}\right)}{u^{\prime}\left(c_{i}\right)}=\frac{1}{1+r}, \quad i=1,2 .
$$

This is the classic condition for optimal intertemporal consumption.

We also have

$$
\frac{\partial \mathcal{L}_{L}}{\partial k}=\pi^{\prime}(k)\left[u\left(c_{2}\right)+v\left(d_{2}\right)-u\left(c_{1}\right)-v\left(d_{1}\right)\right]-\sum_{i} \pi_{i}(k) u^{\prime}\left(c_{i}\right) .
$$

Observe that individuals with $n_{1}$ children have higher disposable incomes, net of cost of children, than individuals with $n_{2}$ children. It follows that $u\left(c_{2}\right)+v\left(d_{2}\right) \leq u\left(c_{1}\right)+$ $v\left(d_{1}\right)$ : utility is higher with $n_{1}$ children than with $n_{2}$. From (3) one then obtains that $\partial \mathcal{L}_{L} / \partial k \leq 0$ and

$$
k_{L}=0 .
$$

That under laissez faire $k=0$, should not be surprising. Children bestow no utility on their parents so that there is no reason to invest in them (given that they are costly to have) ${ }^{7}$

Having characterized the equilibrium under laissez faire, we next characterize the first-best solution for this economy and then turn to the second best.

\section{The utilitarian first-best}

Assume first that the social planner has perfect information, particularly with respect to the individuals' investment levels in children $k$, and that he controls all the relevant variables in the economy: $k, c_{i}, d_{i}$ and $S_{i}$, where $S_{i}$ denotes the savings of the young in state $i=1,2$. He sets these variables to maximize the expected lifetime utility in the steady-state

$$
W=\sum_{i} \pi_{i}(k)\left[u\left(c_{i}\right)+v\left(d_{i}\right)\right]
$$

\footnotetext{
${ }^{7}$ The individual's problem has been set up on the assumption that there are no private insurance markets. If individuals can buy fair insurance, they will pool their resources together and thus maximize their expected utility subject to the single budget constraint

$$
\sum_{i} \pi_{i}(k)\left[y-c_{i}-\frac{d_{i}}{1+r}-k-\theta n_{i}\right] .
$$

In this case, one can easily show that we will continue to have $k=0$, but that $c_{1}=c_{2}$ and $d_{1}=d_{2}$.
} 
subject to the economy's resource constraint,

$$
\sum \pi_{i}(k)\left[y+\frac{S_{i}(1+r)}{\bar{n}(k)}-c_{i}-k-\theta n_{i}-S_{i}-\frac{d_{i}}{\bar{n}(k)}\right]=0
$$

where $\bar{n}(k) \equiv \pi_{1}(k) n_{1}+\pi_{2}(k) n_{2}$, and the young's population size is normalized at one so that there are $1 / \bar{n}(k)$ old people. Our modeling strategy, with a fixed rate of return on savings, $r$, and a non-stochastic aggregate biological rate of return, $\bar{n}$, implies that the choice of storage or PAYGO are, as in Samuelson (1958), mutually exclusive. Expositionally, then, it will be simpler to consider the planner's problem sequentially. First, one finds the optimum conditional on the use of storage and PAYGO; then one compares the levels of welfare achieved at these two conditional optima. This also allows one to simplify the resource constraint (6) and rewrite it as

$$
\sum_{i} \pi_{i}(k)\left[y-c_{i}-\frac{d_{i}}{1+r}-k-n_{i} \theta\right]=0
$$

under storage, and as

$$
\sum_{i} \pi_{i}(k)\left[y-c_{i}-\frac{d_{i}}{\bar{n}(k)}-k-n_{i} \theta\right]=0,
$$

under PAYGO.

\subsection{Storage}

Under the storage technology, the planner maximizes (5) subject to the resource constraint (7). Deriving the first-order conditions of this problem, one can easily establish that $c_{1}=c_{2}=c ; d_{1}=d_{2}=d$. Thus, not surprisingly, consumption levels are equalized across individuals with different number of children. The problem can then be written as

$$
\begin{array}{ll}
\max _{c, d, k} & W_{S}=u(c)+v(d) \\
\text { s.t. } & y-c-\frac{d}{1+r}-k-\bar{n}(k) \theta=0 .
\end{array}
$$

The first-order conditions imply

$$
\frac{v^{\prime}(d)}{u^{\prime}(c)}=\frac{1}{1+r}
$$


and

$$
k^{S}=0 \text {. }
$$

Equation (11) is the optimality condition for intertemporal consumption with the rate of return $r$ on storage. It is also similar to the expression (2) under laissez faire. Condition (12) obtains because $\partial W_{S} / \partial k<0$ (when incorporating the budget constraint). It is identical to condition (4) under laissez faire. ${ }^{8}$ As in there, increasing $k$ has only costs and no benefits. ${ }^{9}$ The values of $c$ and $d$ that solve this problem depend on (the exogenous) value of $r$, as does the associated level of welfare, $W_{S}^{*}(r)$. Moreover, it follows directly from (10) that $W_{S}^{*}$ is an increasing function of $r$.

\subsection{PAYGO}

The problem of the social planner under a PAYGO pension plan is to maximize (5) subject to the resource constraint (8). In this case too, one obtains $c_{1}=c_{2}=c$ and $d_{1}=d_{2}=d$. The Lagrangian expression of the problem can then be written as

$$
\mathcal{L}_{P}=\left[u(c)+v(d)+\mu\left(y-c-\frac{d}{\bar{n}(k)}-k-\bar{n}(k) \theta\right)\right],
$$

with $c, d$ and $k$ as decision variables. One obtains the following optimality conditions ${ }^{10}$

$$
\frac{v^{\prime}(d)}{u^{\prime}(c)}=\frac{1}{\bar{n}(k)}
$$

and

$$
\frac{\partial \mathcal{L}_{P}}{\partial k}=\mu\left[\frac{\bar{n}^{\prime}(k) d}{\bar{n}^{2}}-1-\bar{n}^{\prime}(k) \theta\right]=0 .
$$

Equation (13) is the counterpart of (11) with $\bar{n}(k)-1$ as the net rate of return on "savings". The optimal level of investment in children, $k^{P}$, is determined according to the tradeoff stated in equation (14). This condition requires that the cost of increasing $k$, which includes the extra cost of children $\bar{n}^{\prime}(k) \theta$, equals the benefits associated with

\footnotetext{
${ }^{8}$ The solution is thus identical to that under laissez faire with full private insurance.

${ }^{9}$ This extreme result holds because, as with the laissez faire solution, there are no direct benefits associated with having children in this setting. To capture such benefits, one may include $n$ as a separate argument of the utility function. It would then be possible to have $k^{S}>0$.

${ }^{10}$ The first-order condition with respect to $k$ is for an interior solution. If we have a corner solution $k=0$, the solution is identical to a case with exogenous fertility and one is back to the original Samuelson formulation.
} 
the induced increase in the return to PAYGO. The level of welfare achieved at the PAYGO solution is denoted by $W_{P}^{*}$.

\subsection{PAYGO versus storage}

To determine the first-best solution one must compare the levels of welfare attained at the two conditional optima. First, observe that both conditional solutions imply equalization of consumption levels across parents with different number of children. The parents are thus fully insured for the uncertainty they face over the number of children they will have. Given this common property, it is intuitively obvious that the choice between the technologies must depend solely on their respective "rates of return". Specifically, when $1+r \geq \bar{n}\left(k^{p}\right)$, the storage technology dominates. Its return is at least as high as that of PAYGO, but it does not require the initial investment $k^{P}$. Put differently, the rate of return for PAYGO must be higher than the rate of return on storage to compensate for the investment $k^{P}$. When $1+r<\bar{n}\left(k^{p}\right)$, the choice between the two technologies is more involved. This is best explained using a graphical representation in the $(c, d)$ plane; see Figure 1.

Let $\alpha$ represent the optimal allocation between $c$ and $d$ under PAYGO. It corresponds to a point of tangency between an indifference curve and the resource constraint originating from $y-k^{P}-\theta \bar{n}\left(k^{p}\right)$ (endowment minus total cost of children) with a slope (in absolute value) of $\bar{n}\left(k^{P}\right)$. Define $\hat{r}$ as the rate of return on storage at which first-best welfare level under storage equals its PAYGO level: $W_{S}^{*}(\hat{r})=W_{P}^{*}$. Graphically, $1+\hat{r}$ is the slope of the budget line (under storage) with horizontal intercept of $y-\theta \bar{n}(0)$ and which is tangent to the indifference curve corresponding to $W_{P}^{*} \cdot{ }^{11}$ One attains the same

\footnotetext{
${ }^{11}$ The graphical representation, and the comparison between $1+r$ and $\bar{n}\left(k^{P}\right)$, assume no initial fixed investment costs in the storage technology. This is inconsequential; its only potential effect would be to possibly change the direction of the inequality $\hat{r}<\bar{n}\left(k^{P}\right)-1$ (the critical mechanism-switching rate of return on storage is less than the PAYGO rate of return) which Figure 1 displays. But this is all that it may do. The important point is that none of our conclusions rests on the direction of this inequality.

To see why the direction of the inequality may change, let $k^{S}$ denote the cost of investment in storage. This changes the position of $y-\theta \bar{n}(0)$ intercept to $y-k^{S}-\theta \bar{n}(0)$. No further modification is called for as long as $k^{S}+\theta \bar{n}(0)<k^{P}+\theta \bar{n}\left(k^{P}\right)$. On the other hand, if $k^{S}+\theta \bar{n}(0)>k^{P}+\theta \bar{n}\left(k^{P}\right)$, the $y-k^{S}-\theta \bar{n}(0)$ intercept will be to the left of $y-k^{P}-\theta \bar{n}\left(k^{P}\right)$ intercept so that the line that originates from $y-k^{S}-\theta \bar{n}(0)$, and is tangent to the indifference curve corresponding to $W_{P}^{*}$ at $\beta$, will have a steeper slope in absolute value than $\bar{n}\left(k^{P}\right)$ (the absolute value of the line through $y-k^{P}-\theta \bar{n}\left(k^{P}\right)$ and tangent to the same indifference curve). In this case, $\beta$ will be above and to the left of $\alpha$. Consequently,
} 


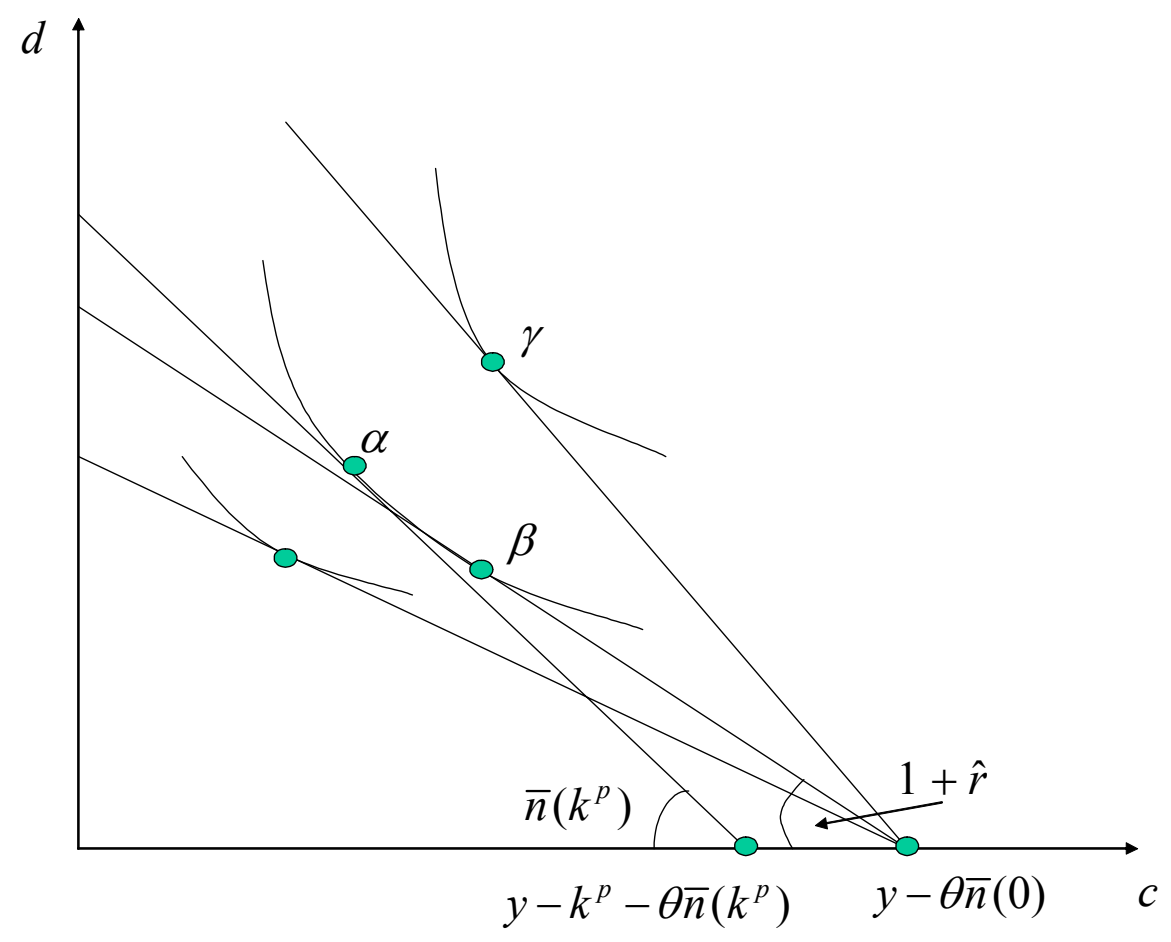

Figure 1: Optimal allocations with storage and PAYGO technologies

utility level under storage at point $\beta$, with more first-period and less second-period consumption. Observe also that $\beta$ is not available unless everyone is subjected to PAYGO. That is, it is not optimal to use the two mechanisms simultaneously. With $r>\hat{r}$, society would opt for the storage technology and the solution $\gamma$. With $r<\hat{r}$, the demographic mechanism dominates storage.

\subsection{Decentralization of the optimum}

We now briefly examine how the first-best optimum can be decentralized. With the firstbest consumption levels (in both periods) being independent of the number of children, one may think of the decentralized solution as offering individuals full insurance against the risk (from a personal perspective) of having many children. When the storage one chooses PAYGO whenever $1+r \leq \bar{n}\left(k^{P}\right)$. Otherwise, if $1+r>\bar{n}\left(k^{P}\right)$, one will opt for one or the other mechanism depending on how $r$ compares with $\hat{r}$. Put differently, one must have $\hat{r}>\bar{n}\left(k^{P}\right)-1$. 
technology dominates PAYGO, it suffices to compensate those with $n_{2}$ children for their extra cost of raising children. This is achieved by levying a lump-sum tax equal to $\pi(0)\left(n_{2}-n_{1}\right) \theta$ on parents with $n_{1}$ children, and giving $(1-\pi(0))\left(n_{2}-n_{1}\right) \theta$ to parents who have $n_{2}$ children. Such a tax and transfer policy fully insures the parents, who will now face a net cost (inclusive of the tax and transfer) of $\theta \bar{n}(0)$ in raising their children regardless of their number, while satisfying the government's budget constraint. ${ }^{12}$

When PAYGO dominates storage, decentralization is somewhat more intricate. First, in order for the payroll taxes $\left(T_{i}^{\prime}\right.$ 's) and pension benefits $\left(P_{i}\right.$ 's) to satisfy the government's budget constraint, and with $\bar{n}(k)$ young individuals for every old person, we must have $\bar{n}(k) \sum \pi_{i}(k) T_{i}=\sum \pi_{i}(k) P_{i}$. Second, as with the storage technology, the individualized payroll taxes must satisfy $T_{1}-T_{2}=\left(n_{2}-n_{1}\right) \theta$ in order to equalize first-period consumption levels. Third, to equalize second-period consumption levels, pensions will have to be independent of the number of children: $P_{1}=P_{2}=P$. Finally, we also need to induce the "correct" level of investment in children and ensure that there are no private savings.

If there are no benefits associated with investment in children, no one will choose a positive level of $k$. To ensure some investment, pensions must in part be conditioned on $k$. Specifically, let $k^{P}$ and $d^{P}$ denote the (PAYGO) first-best values of $k$ and $d$. Set $P=\bar{P}+k \bar{n}\left(k^{P}\right)$ where $\bar{P}$ is fixed and satisfies $\bar{P}+k^{P} \bar{n}\left(k^{P}\right)=d^{P}$. It is easy to show that under this pension scheme, and with the "appropriate" choice of $T_{1}$ and $T_{2}$, all individuals would choose $k=k^{P}$, opt for zero private savings (negative savings are not allowed) and choose $d^{P}$ and $c^{P}$ as well ( $c^{P}$ is the PAYGO first-best value of $c$ ). A subsidy on $k$ at the rate of $\bar{n}\left(k^{P}\right)-1$ per unit of $k$ (to ensure a gross return of $\bar{n}\left(k^{P}\right)$ ) is necessary because each individual ignores the atomistic impact of his choice of $k$ on aggregate $k$ and thus on $\bar{n}(k)$, the rate of return of the PAYGO system that each person enjoys. Put differently, an individual's investment in children creates a positive externality in that it increases the number of future working persons who will be supporting a retired person. ${ }^{13}$ Consequently, unless there is a (Pigouvian) subsidy, individuals will set $k$ at

\footnotetext{
${ }^{12}$ Recall that there are $(1-\pi(0))$ parents with $n_{1}$ children, and $\pi(0)$ with $n_{2}$ children, so that $(1-\pi(0)) T_{1}+\pi(0) T_{2}=0$.

${ }^{13}$ This is what the literature refers to as the "intergenerational transfer effect." It is the only effect
} 
zero and there will be no investment in children.

Observe that the Pigouvian subsidy is set at a rate that brings the private cost of investing in $k$ equal to its social cost which is one. To see this, recall that in the above scheme the subsidy on $k$ is paid in the second period as part of one's pension benefits. Discounted to the first period (with a discount rate of $1 / \bar{n}\left(k^{P}\right)$ ), its value will be equal to 1 - the marginal cost of $k$. Observe also that, at the optimal solution, the marginal cost of $k$ equals the marginal (net) social benefits of $k$. One can see this by rewriting (14) as

$$
1=\frac{\bar{n}^{\prime}\left(k^{P}\right) d^{P}}{\left[\bar{n}\left(k^{P}\right)\right]^{2}}-\bar{n}^{\prime}\left(k^{P}\right) \theta,
$$

where the right-hand side of this expression is the net social marginal benefit of $k$. Its first terms measures the induced impact on the return of PAYGO, while the second terms represents the extra cost of raising children.

We summarize the results of this section as

Proposition 1 (i) The first-best allocation under storage requires that parents do not invest in fertility, have equal consumption levels regardless of their number of children in both periods of their lives, and that their first- and second-period consumption levels satisfy the classic condition for intertemporal consumption at a rate of return determined by the storage technology. The allocation can be decentralized by levying a lump-sum tax equal to $\pi(0)\left(n_{2}-n_{1}\right) \theta / 2$ on parents with $n_{1}$ children, and giving $(1-\pi(0))\left(n_{2}-n_{1}\right) \theta / 2$ to each parent who has $n_{2}$ children.

(ii) The first-best allocation under PAYGO requires that parents make an investment in fertility equal to $k^{p}$, the solution to equation (14), have equal consumption levels regardless of the number of their children in both periods of their lives, and that their first and second period consumption levels satisfy the condition for intertemporal consumption at the rate of $\bar{n}\left(k^{P}\right)$. The allocation can be decentralized by linking pension benefits, $P$,

associated with increasing fertility in our Samuelsonian setup with a storage technology. If, on the other hand, the technology for transferring resources across generations is a neoclassical production function as in Diamond (1965), there will also be a cost associated with investing in fertility which results in a negative externality. This is the required expansion in capital in order to maintain the same capital output ratio in the face of an expanding work force as the fertility increases. This is termed in the literature "the capital dilution effect." In the presence of this latter effect, the net externality associated with an increase in fertility is not necessarily positive. 
to investment in children according to $P=\bar{P}+k \bar{n}\left(k^{P}\right)$ where $\bar{P}$ is fixed and satisfies $\bar{P}+k^{P} \bar{n}\left(k^{P}\right)=d^{P}$ (the superscript $P$ denotes a PAYGO first-best value), coupled with individualized payroll taxes that satisfy $T_{1}-T_{2}=\left(n_{2}-n_{1}\right) \theta>0$ and the government's per period budget constraint, $\sum \pi_{i}(k) T_{i}=\sum \pi_{i}(k) P_{i} / \bar{n}(k)$.

(iii) Let $\hat{r}$ denote the rate of return on storage at which first-best welfare level under storage equals its PAYGO level. Then, for all $r<\hat{r}, P A Y G O$ dominates storage and for all $r>\hat{r}$, storage dominates PAYGO.

\section{Second-best solution}

The first-best characterization rests on the assumption that the government can control $k$ fully, either directly or through a subsidy. This will be the case if $k$ (and thus $c$ ) are publicly observable. When the observability assumption is not satisfied, one will be in a second-best environment. Under this circumstance, the full insurance prescriptions of a first-best world may not hold. We shall examine this question below.

Assume that $k$ and $c$ are not publicly observable but $n_{i}$ 's $(i=1,2)$ are. Public policy consists of either a tax system while letting the young save for their own retirement; ${ }^{14}$ or a pension plan through which the government collects taxes from the current young and distributes the proceeds to the retired. With $n_{i}$ 's being publicly observable, taxes $T_{i}$ 's and transfers $P_{i}$ 's may be conditioned on the number of children. Whether or not this should be the case is an interesting policy question which this section attempts to shed light on. Next section studies the setting where contributions are required by law to be uniform.

\subsection{Storage}

Under the storage technology, the resource constraint is given by

$$
\sum_{i} \pi_{i}(k)\left[y-c_{i}-\frac{d_{i}}{1+r}-k-n_{i} \theta\right]=0 .
$$

This is identical to the first-best constraint. Most significantly, the unobservability of $k$ is of no relevance here. In particular, there is no reason why individuals should

\footnotetext{
${ }^{14}$ Alternatively, the government may institute a fully-funded pension plan, taxing away all savings, investing (storing) the proceeds and distributing the investments and the returns as pensions.
} 
be induced to choose a different level of $k$ than they would do otherwise. Indeed, the (conditional) first-best allocation of subsection 3.4 (which requires equalization of consumptions levels for parents with different number of children) is attainable as long as tax payments are not restricted to be uniform. As in the first-best, this is done through levying a lump-sum tax equal to $\pi(0)\left(n_{2}-n_{1}\right) \theta$ on parents with $n_{1}$ children, and giving $(1-\pi(0))\left(n_{2}-n_{1}\right) \theta$ to parents who have $n_{2}$ children. The investment level $k$ is set at zero which is in line with individual incentives. Summing up, with storage and state-dependant contributions, the (conditional) first- and second-best solutions coincide.

\subsection{PAYGO}

Recall that the first-best outcome entailed two properties: full insurance plus an optimal choice of $k$ such that, given an exogenous rate of return on storage equal to $r, \bar{n}\left(k^{p}\right)>$ $1+\hat{r}>1+r$. With $k$ being directly "under control," the planner could set it at its optimal level without one having to forgo the full insurance property. In a second best environment, $k$ can no longer be directly controlled and keeping the full insurance property is consistent with $k=0$ only. Second-best optimality may then require trading off the full insurance property for a positive choice of $k$ (although not at its first-best value). We will see below this is precisely the outcome when one can control $k$ only indirectly, through the incentives that the pension scheme provides.

The young's problem, when facing the policy instruments $T_{1}, T_{2}, P_{1}$ and $P_{2}$, is

$$
\begin{array}{rlrl}
\max _{c_{1}, c_{2}, k} & U & =(1-\pi(k))\left[u\left(c_{1}\right)+v\left(d_{1}\right)\right]+\pi(k)\left[u\left(c_{2}\right)+v\left(d_{2}\right)\right], \\
\text { s.t. } & c_{i} & =y-k-T_{i}-\theta n_{i} \quad i=1,2, \\
& d_{i} & =P_{i} \quad i=1,2 .
\end{array}
$$

This yields the following first-order condition for an interior solution for $k$,

$$
\pi^{\prime}(k)\left\{u\left(c_{2}\right)+v\left(d_{2}\right)-\left[u\left(c_{1}\right)+v\left(d_{1}\right)\right]\right\}-(1-\pi(k)) u^{\prime}\left(c_{1}\right)-\pi(k) u^{\prime}\left(c_{2}\right)=0 .
$$

Naturally, the second-order condition $\left.\Delta \equiv\left(d^{2} U / d k^{2}\right)\right|_{k=\widetilde{k}}<0$ must also be satisfied; we 
shall assume throughout the paper that this is the case. ${ }^{15}$

The first term on the left-hand side of (19) measures the benefit (for the individual) of increasing $k$, while the second term measures the cost. Not surprisingly, an interior solution requires marginal benefits to equal marginal costs. Observe that when the left-hand side of (19) is non-positive at $k=0$, we have a corner solution and the individual does not invest in $k$. This occurs for instance when $c_{1}=c_{2}$ (i.e., when $\left.T_{1}-T_{2}=\theta\left(n_{2}-n_{1}\right)\right)$ and $d_{1}=d_{2}$. The solution to the individual's problem, denoted by $\widetilde{k}\left(T_{1}, T_{2}, P_{1}, P_{2}\right)$, describes all possible values of $k$ that the government can induce through its choice of $T_{1}, T_{2}, P_{1}$ and $P_{2}$. The following lemma establishes the comparative static properties of $\widetilde{k}\left(T_{1}, T_{2}, P_{1}, P_{2}\right)$, which will prove useful in studying the government's problem.

Lemma 1 If $\widetilde{k}\left(T_{1}, T_{2}, P_{1}, P_{2}\right)$ is given by an interior solution,

$$
\begin{aligned}
\frac{\partial \widetilde{k}}{\partial T_{1}} & =\frac{\pi^{\prime}(k) u^{\prime}\left(c_{1}\right)+[1-\pi(k)] u^{\prime \prime}\left(c_{1}\right)}{(-\Delta)} \lessgtr 0 \\
\frac{\partial \widetilde{k}}{\partial T_{2}} & =\frac{-\pi^{\prime}(k) u^{\prime}\left(c_{2}\right)+\pi(k) u^{\prime \prime}\left(c_{2}\right)}{(-\Delta)}<0 \\
\frac{\partial \widetilde{k}}{\partial P_{1}} & =\frac{-\pi^{\prime}(k) v^{\prime}\left(d_{1}\right)}{(-\Delta)}<0 \\
\frac{\partial \widetilde{k}}{\partial P_{2}} & =\frac{\pi^{\prime}(k) v^{\prime}\left(d_{2}\right)}{(-\Delta)}>0 .
\end{aligned}
$$

At a corner solution, $k=0$ and all partial derivatives of $\widetilde{k}$ are also equal to zero. ${ }^{16}$

The inequality signs are as expected. The ambiguity of the first derivative is due to the conflicting income and incentive effects of increasing $T_{1}$. For simplicity we shall concentrate on the "normal" case which occurs if $\partial \widetilde{k} / \partial T_{1}>0$.

\footnotetext{
${ }^{15}$ We have

$$
\Delta=\frac{\pi^{\prime \prime}(k)}{\pi^{\prime}(k)} \sum_{i} \pi_{i}(k) u^{\prime}\left(c_{i}\right)+2 \pi^{\prime}(k)\left[u^{\prime}\left(c_{1}\right)-u^{\prime}\left(c_{2}\right)\right]+\sum_{i} \pi_{i}(k) u^{\prime \prime}\left(c_{i}\right) .
$$

The first and the last expressions in the right-hand side of above are negative due to concavity of $u($. and $\pi($.$) Consequently, as long as c_{2}$ does not exceed $c_{1}$ by "much", $\Delta$ will be negative.

${ }^{16} \mathrm{At}$ the transition between these two regimes $\widetilde{k}$ may not be differentiable (even though it is continuous as long as the second-order condition holds). We ignore this technical difficulty for the sake of simplicity.
} 
Consider now the government problem. A first ingredient is the resource constraint. With $\bar{n}(k)$ young individuals for every old person, this is given by

$$
\bar{n}(k)\left[(1-\pi(k)) T_{1}+\pi(k) T_{2}\right]=(1-\pi(k)) P_{1}+\pi(k) P_{2} .
$$

Equation (24) is a rewriting of (8) in terms of the second-best policy instruments. It requires that total contributions equal total pension benefits. A second element is the constraint that $k=\widetilde{k}\left(T_{1}, T_{2}, P_{1}, P_{2}\right)$ which reflects the government's indirect control of the level of investment in children. The conditional second-best problem for the PAYGO case is then summarized by the Lagrangian

$$
\begin{aligned}
\Gamma^{S} & =(1-\pi(k))\left[u\left(c_{1}\right)+v\left(d_{1}\right)\right]+\pi(k)\left[u\left(c_{2}\right)+v\left(d_{2}\right)\right] \\
& +\mu\left\{(1-\pi(k))\left(\bar{n}(k) T_{1}-P_{1}\right)+\pi(k)\left(\bar{n}(k) T_{2}-P_{2}\right)\right\} \\
& +\eta\left[\widetilde{k}\left(T_{1}, T_{2}, P_{1}, P_{2}\right)-k\right]
\end{aligned}
$$

where $d_{i}=P_{i}$ and $c_{i}=y-k-T_{i}-\theta n_{i}(i=1,2)$. The first-order conditions are ${ }^{17}$

$$
\begin{aligned}
\frac{\partial \Gamma^{S}}{\partial T_{1}} & =[1-\pi(k)]\left[\mu \bar{n}(k)-u^{\prime}\left(c_{1}\right)\right]+\eta \frac{\partial \widetilde{k}}{\partial T_{1}}=0, \\
\frac{\partial \Gamma^{S}}{\partial T_{2}} & =\pi(k)\left[\mu \bar{n}(k)-u^{\prime}\left(c_{2}\right)\right]+\eta \frac{\partial \widetilde{k}}{\partial T_{2}}=0, \\
\frac{\partial \Gamma^{S}}{\partial P_{1}} & =(1-\pi(k))\left[v^{\prime}\left(d_{1}\right)-\mu\right]+\eta \frac{\partial \widetilde{k}}{\partial P_{1}}=0, \\
\frac{\partial \Gamma^{S}}{\partial P_{2}} & =\pi(k)\left[v^{\prime}\left(d_{2}\right)-\mu\right]+\eta \frac{\partial \widetilde{k}}{\partial P_{2}}=0, \\
\frac{\partial \Gamma^{S}}{\partial k} & =\mu\left\{-\pi^{\prime}(k)\left(\bar{n}(k) T_{1}-d_{1}\right)+(1-\pi(k)) T_{1}\left(n_{2}-n_{1}\right) \pi^{\prime}(k)\right. \\
& \left.+\pi^{\prime}(k)\left(\bar{n}(k) T_{2}-d_{2}\right)+\pi(k) T_{2}\left(n_{2}-n_{1}\right) \pi^{\prime}(k)\right\}-\eta=0 .
\end{aligned}
$$

Recall that the definition of $\widetilde{k}\left(T_{1}, T_{2}, P_{1}, P_{2}\right)$ encompasses both interior as well as corner solutions (of the individuals' problem). We start by considering the case in which the optimal policy induces an interior solution for $\widetilde{k}$. The case of $k=0$ will be discussed later.

\footnotetext{
${ }^{17}$ In calculating $\partial \Gamma^{S} / \partial k$, we have utilized the individual's first-order condition (19).
} 


\subsubsection{Interior solution for $\widetilde{k}$}

The first-order conditions (25)-(29) indicate that the properties of the second-best solution depend crucially on the sign of $\eta$. To the extent that $k$ entails a positive externality so that the individuals tend to choose a level of $k$ that is "too low", one would expect $\eta>0$. The following lemma shows that this is effectively the case, as long as $\partial \widetilde{k} / \partial T_{1} \geq 0$ holds.

Lemma 2 If $\partial \widetilde{k} / \partial T_{1} \geq 0$, then $\eta>0$.

Proof. The proof is by contradiction. Assume $\eta \leq 0$. Then the first-order conditions (27)-(28), together with the concavity of $v(\cdot)$, imply $d_{2} \leq d_{1}$. Similarly, (25)-(26), the assumption that $\partial \widetilde{k} / \partial T_{1} \geq 0$ and the concavity of $u(\cdot)$ result in $c_{2} \leq c_{1}$. Given these two inequalities, it follows directly from (19) that one cannot have an interior solution for $\widetilde{k}$, and we have a contradiction.

We are now in a position to study the properties of the second-best solution. We are particularly interested in the relationship between payroll taxes and pension benefits on the one hand, and the number of children on the other. Consider the benefits first. With $\eta>0$, it follows from (22) and (27) that $v^{\prime}\left(d_{1}\right)-\mu>0$, and from (23) and (28) that $v^{\prime}\left(d_{2}\right)-\mu<0$. The concavity of $v($.$) then implies d_{2}>d_{1}$. Regarding payroll taxes, with $\partial \widetilde{k} / \partial T_{1} \geq 0$, expression (21) and equations (25)-(26) yield $\mu \bar{n} \leq u^{\prime}\left(c_{1}\right)$ and $\mu \bar{n}>u^{\prime}\left(c_{2}\right)$. Concavity of $u($.$) then implies c_{2}>c_{1}$, so that $T_{1}-T_{2}>\theta\left(n_{2}-n_{1}\right)>0$.

The following proposition summarizes the second-best results under storage and PAYGO.

Proposition 2 (a) Under storage the (conditional) first- and second-best solutions coincide.

(b) Assume an increase in payroll taxes on parents with small number of children increases (or leaves unchanged) their investment in children $\left(\partial \widetilde{k} / \partial T_{1} \geq 0\right)$. Then in the second-best allocation under a PAYGO pension system: Benefits should increase with the number of children $\left(P_{2}>P_{1}\right)$; payroll taxes must decrease with the number of children; families with a higher number of children are more than compensated for the extra cost 
of children $\left(T_{1}-T_{2}>\theta\left(n_{2}-n_{1}\right)>0\right.$, and $\left.c_{2}>c_{1}\right)$; the investment in children, and the resulting average fertility rate, are less than their corresponding first-best levels.

To interpret these results, recall that the first-best solution requires full insurance: consumption levels of the young and the retired are independent of the number of children. In a first-best setting, this is provided without preventing $k$ to also be set at its optimal level. As shown earlier, one could induce an optimal level of $k$, a publicly observable variable, by linking pension benefits to it. When $k$ is not observable, this procedure is no longer feasible. Instead, pension benefits, and contributions, may be linked to the number of children which are observable, and whose realization can be influenced by $k$.

Specifically, if contributions and benefits entail full insurance, individuals will have no incentive to invest in children and $k=0$. To induce a positive $k$, contributions and/or benefits must be linked to the number of children. In consequence, one loses the full insurance property. The optimal policy then strikes a balance between insurance and incentive considerations. Roughly speaking, if one were to think of $k$ as effort, we have a moral hazard problem which calls for less-than-full insurance. It is thus not surprising that $d_{2}>d_{1}$ and $c_{2}>c_{1}$. The higher consumption levels for parents with a greater number of children, works as an incentive mechanism to induce positive investment in children. Observe that $c_{2}>c_{1}$ does not just require $T_{1}>T_{2}$, it calls for the stronger condition $T_{1}-T_{2}>\theta\left(n_{2}-n_{1}\right)$. In words, parents with a higher number of children should see their taxes reduced by an amount that exceeds the extra costs they incur as a result of having more children. This may appear surprising at first, but it is easily understood by realizing that when $T_{1}-T_{2} \leq \theta\left(n_{2}-n_{1}\right)$, one can gain on both the insurance and incentive fronts by widening the gap between $T_{1}$ and $T_{2}$.

As a final observation, manipulate the first-order conditions (25)-(28) to arrive at

$$
\frac{(1-\pi(k)) v^{\prime}\left(d_{1}\right)+\pi(k) v^{\prime}\left(d_{2}\right)}{(1-\pi(k)) u^{\prime}\left(c_{1}\right)+\pi(k) u^{\prime}\left(c_{2}\right)}=\frac{1-\frac{\eta}{\mu}\left(\frac{\partial \widetilde{k}}{\partial P_{1}}+\frac{\partial \widetilde{k}}{\partial P_{2}}\right)}{\bar{n}(k)+\frac{\eta}{\mu}\left(\frac{\partial \widetilde{k}}{\partial T_{1}}+\frac{\partial \widetilde{k}}{\partial T_{2}}\right)}
$$

The left-hand side of equation (30) denotes the marginal rate of substitution between $d_{i}$ 's and $c_{i}$ 's. This was set equal to equal to $1 / \bar{n}(k)$, their relative "marginal costs", 
under first best; see equation (13). Consequently, the second best implies that the individuals' life-cycle consumption patterns are distorted. If the difference in the young's consumption levels is "close" to the difference in the old's consumption levels (between people with different number of children),${ }^{18}$ the right-hand side of (30) is greater than $1 / \bar{n}(k)$. This means that the marginal rate of substitution between $d_{i}$ 's and $c_{i}$ 's increases as one goes from first best to second best. Put differently, less resources are transferred to the future for consumption in the second best relative to first best.

\subsubsection{Corner solution at $\widetilde{k}=0$}

In describing an individual's behavior facing the policy instruments $T_{1}, T_{2}, P_{1}$ and $P_{2}$ under PAYGO, we pointed out that if the individual is induced to choose $c_{1}=c_{2}$ and $P_{1}=P_{2}$, then he will opt for $k=0$. Similarly, one can deduce from the first-order conditions (25)-(28) of the second-best problem, that if $k=0$ is the second-best choice of $k$, optimality requires $c_{1}=c_{2}$ and $P_{1}=P_{2}$. Consequently, one faces the possibility of having $c_{1}=c_{2}, d_{1}=d_{2}$ and $k=0$ as the second-best solution. To investigate this possibility, evaluate $\partial \Gamma^{S} / \partial k$ at $c_{1}=c_{2}=c, d_{1}=d_{2}=d, k=0$ and simplify. ${ }^{19}$ We have

$$
\frac{\partial \Gamma^{S}}{\partial k}=-u^{\prime}(c)+\mu \pi^{\prime}(0)\left(n_{2}-n_{1}\right)\left[\frac{d}{\bar{n}(0)}-\theta \bar{n}(0)\right]
$$

$$
\begin{aligned}
& \frac{1-\frac{\eta}{\mu}\left(\frac{\partial \widetilde{k}}{\partial P_{1}}+\frac{\partial \widetilde{k}}{\partial P_{2}}\right)}{\bar{n}(k)+\frac{\eta}{\mu}\left(\frac{\partial \widetilde{k}}{\partial T_{1}}+\frac{\partial \widetilde{k}}{\partial T_{2}}\right)}-\frac{1}{\bar{n}(k)} \\
& =\frac{-\frac{\eta}{\mu}\left\{\pi^{\prime}(k)\left[u^{\prime}\left(c_{1}\right)-u^{\prime}\left(c_{2}\right)\right]+\pi^{\prime}(k) \bar{n}(k)\left[v^{\prime}\left(d_{2}\right)-v^{\prime}\left(d_{1}\right)\right]+\sum \pi_{i} k u^{\prime \prime}\left(c_{i}\right)\right\}}{(-\Delta) \bar{n}(k)+\left[\bar{n}(k)+\frac{\eta}{\mu}\left(\frac{\partial \widetilde{k}}{\partial T_{1}}+\frac{\partial \tilde{k}}{\partial T_{2}}\right)\right]} .
\end{aligned}
$$

The denominator of this expression is positive. To see this, observe that the property $d_{2}>d_{1}$ (which follows from our assumption that $\left.\partial \widetilde{k} / \partial T_{1}>0\right)$, coupled with the concavity of $v($.$) , imply$

$$
\frac{\partial \widetilde{k}}{\partial P_{1}}+\frac{\partial \widetilde{k}}{\partial P_{2}}=\frac{\pi^{\prime}(k)\left[v^{\prime}\left(d_{2}\right)-v^{\prime}\left(d_{1}\right)\right]}{(-\Delta)}<0 .
$$

It then follows from equation (30) that

$$
\bar{n}(k)+\frac{\eta}{\mu}\left(\frac{\partial \widetilde{k}}{\partial T_{1}}+\frac{\partial \widetilde{k}}{\partial T_{2}}\right)>0 .
$$

The numerator will also be positive as long as $u^{\prime}\left(c_{1}\right)-u^{\prime}\left(c_{2}\right)>0$ is "close," in absolute value, to $\bar{n}(k)\left[v^{\prime}\left(d_{2}\right)-v^{\prime}\left(d_{1}\right)\right]<0$.

${ }^{19}$ The expression for $\partial \Gamma^{S} / \partial k$ differs from that given by (29) because the latter was derived assuming an interior solution for $\tilde{k}$. 
One can see from this expression that $\partial \Gamma^{S} / \partial k$ may take a negative value at $k=0$. Thus we cannot a priori rule out a solution with $d_{1}=d_{2}, c_{1}=c_{2}$ and $k=0$. This would be the case if $\pi$ is not very responsive to $k$ (so that $\pi^{\prime}(0)$ is close to zero), if $\theta$ is "large", or if individuals have a very large degree of risk aversion. ${ }^{20}$ If this happens, the tradeoff between $c$ and $d$ will again be at its first-best value of $1 / \bar{n}(k)$, albeit at $k=0$. [See equation (30) which would then simplify to (13)]. The solution is effectively the same as the first-best outcome under storage, with a rate of return equal to $(1+r)=\bar{n}(0)$.

\subsection{PAYGO versus storage in the second best}

Denote the welfare achieved at the second-best solution by $W_{P}^{S B}$ (for PAYGO) and $W_{S}^{S B}(r)$ (for storage). As in the first best, the choice between the two mechanisms hinges on the exogenous level of $r$. The results obtained in subsections 4.2 and 4.1 imply that $W_{P}^{S B} \leq W_{P}^{*},{ }^{21}$ while $W_{S}^{S B}(r)=W_{S}^{*}(r)$ holds for any level of $r$. Thus, under PAYGO, the unobservability of $k$ results in a welfare loss. Under storage, on the other hand, the first-best outcome is achieved even if $k$ is unobservable. Let $r^{S B}$ denote the critical level of return satisfying $W_{S}^{S B}\left(r^{S B}\right)=W_{P}^{S B}<W_{P}^{*}$. It then immediately follows that $r^{S B}<\widehat{r}$, where $\widehat{r}$ is the critical level in the first best. In other words, whenever storage is optimal in the first best, it is also optimal in the second best. On the other hand, when PAYGO is optimal in the first best, it may or may not be optimal in the second best. Put differently, the range of values of $r$ for which storage dominates PAYGO is larger in the second best than in the first best. This is depicted in Figure 2. ${ }^{22}$

We end this section with another proposition.

Proposition 3 Let $r^{S B}$ denote the rate of return on storage at which second-best welfare level under storage equals its PAYGO level $\left[W_{S}^{S B}\left(r^{S B}\right)=W_{P}^{S B}\right]$. We have: $r^{S B}<\hat{r}$,

\footnotetext{
${ }^{20}$ With a large degree of risk aversion, individuals would want to set $c_{1}=c_{2}$ and $d_{1}=d_{2}$. It then follows from the young's optimization problem (16)-(18) that they will set $k=0$.

${ }^{21}$ The equality sign applies if the first- and second-best outcomes under PAYGO are given by the corner solution $k=0$, and $c_{1}=c_{2}, d_{1}=d_{2}$.

${ }^{22}$ Observe also that a simple graphical representation as in Figure 1 can no longer be provided because, in the second best under PAYGO, $c_{1} \neq c_{2}$ and $d_{1} \neq d_{2}$. The exception is when the PAYGO second best implies a corner solution for $k=0$. We would then have, for both mechanisms, budget lines starting from $y$ and it will be sufficient to compare $r$ with $\bar{n}(0)$.
} 


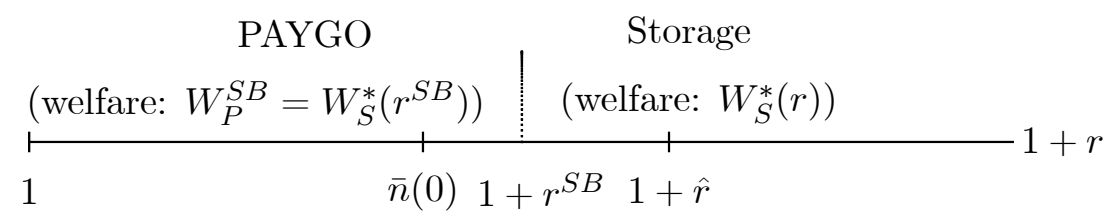

Figure 2: PAYGO versus storage in the second best.

$W_{S}^{S B}\left(r^{S B}\right)=W_{S}^{*}\left(r^{S B}\right)$, and $W_{P}^{S B}<W_{P}^{*}[\hat{r}$ is the welfare-equalizing rate under first best, and $*$ indicates first-best values.]

\section{Second best PAYGO with state-independent first-period taxes}

The discussion thus far has allowed for tax and benefit schemes that are both state dependent. As a policy prescription, however, one may want to restrict pension contributions and/or pension benefits to be independent of the number of children. Indeed, depending on the timing of the decision process, there are circumstances under which differentiation of $T_{1}$ from $T_{2}$ may not even be possible. ${ }^{23}$ With this in mind, we shall now discuss a special case of our model where the tax payments do not vary with the number of children. This setting too constitutes a departure from the traditional PAYGO pension plans under which it is not just the taxes on the young, but also the pension benefits of the old, that are invariant to the number of children.

Formally, observe first that the equality of first-period tax payments implies, from $(17)$,

$$
c_{1}-c_{2}=\theta\left(n_{2}-n_{1}\right)>0
$$

Thus, individuals who end up with more children would also have to pay in full the corresponding additional costs (this is in addition to $k$ paid by everyone). Now, as far as the individuals are concerned, they face the same optimization problem as before which would then yield a first-order condition identical to (19). ${ }^{24}$ The solution for $k$

\footnotetext{
${ }^{23}$ This will be the case, for example, if taxes are levied before $n$ is realized.

${ }^{24}$ With $T_{1}=T_{2}, c_{1}-c_{2}=\theta\left(n_{2}-n_{1}\right)>0$ so that $u^{\prime}\left(c_{1}\right)<u^{\prime}\left(c_{2}\right)$. It then follows from the expression
} 
will depend on $P_{1}, P_{2}$ and $T\left(=T_{1}=T_{2}\right)$, with the expressions for $\partial \widetilde{k} / \partial P_{1}$ and $\partial \widetilde{k} / \partial P_{2}$ remaining unchanged from (22)-(23). One can also easily show that, with $c_{1}>c_{2}$, $\partial \widetilde{k} / \partial T=\partial \widetilde{k} / \partial T_{1}+\partial \widetilde{k} / \partial T_{2}<0$.

Regarding the government's optimization, one must now impose an additional constraint $\left(T_{1}=T_{2}=T\right)$ on the second-best problem. This is summarized by the Lagrangian

$$
\begin{aligned}
\Gamma^{C} & =(1-\pi(k))\left[u\left(c_{1}\right)+v\left(d_{1}\right)\right]+\pi(k)\left[u\left(c_{2}\right)+v\left(d_{2}\right)\right] \\
& +\mu\left\{(1-\pi(k))\left(\bar{n}(k) T_{1}-P_{1}\right)+\pi(k)\left(\bar{n}(k) T_{2}-P_{2}\right)\right\} \\
& +\eta\left[\widetilde{k}\left(T_{1}, T_{2}, P_{1}, P_{2}\right)-k\right]+\lambda\left(T_{1}-T_{2}\right),
\end{aligned}
$$

with $d_{1}=P_{1}$ and $d_{2}=P_{2}$. The first-order conditions with respect to $P_{1}, P_{2}$ and $k$, are as in the unconstrained case. As in that case, we will again have, as long as the solution for $\widetilde{k}$ is interior, $\eta>0$ and $d_{2}>d_{1}$. Interestingly, these results now hold regardless of the sign of $\partial \widetilde{k} / \partial T_{1} .{ }^{25}$ Of course, the levels of $d_{1}, d_{2}$ and $k$ will be different as $c_{1}$ now exceeds $c_{2}$ rather than the other way around.

To see another implication of the $T_{1}=T_{2}$ constraint, assume again that $\partial \widetilde{k} / \partial T_{1} \geq 0$. Under this circumstance, one can show that $\lambda<0$. Thus reducing $T_{2}$ and increasing $T_{1}$ from their current equal value are welfare improving. Finally, corresponding to equation (30) under the unconstrained second best, we now have

$$
\frac{(1-\pi(k)) v^{\prime}\left(d_{1}\right)+\pi(k) v^{\prime}\left(d_{2}\right)}{(1-\pi(k)) u^{\prime}\left(c_{1}\right)+\pi(k) u^{\prime}\left(c_{2}\right)}=\frac{1-\frac{\eta}{\mu}\left(\frac{\partial \widetilde{k}}{\partial P_{1}}+\frac{\partial \widetilde{k}}{\partial P_{2}}\right)}{\bar{n}(k)+\frac{\eta}{\mu}\left(\frac{\partial \widetilde{k}}{\partial T}\right)}>\frac{1}{\bar{n}(k)},
$$

where the inequality sign follows from the fact that $\partial \widetilde{k} / \partial T=\partial \widetilde{k} / \partial T_{1}+\partial \widetilde{k} / \partial T_{2}<0$, and $\partial \widetilde{k} / \partial P_{1}+\partial \widetilde{k} / \partial P_{2}<0 .{ }^{26}$ This duplicates the result under the unrestricted second best. However, there, we had to assume that the differences in consumption levels between people with different number of children were "close" for when they are young and when for $\Delta$ in footnote 15 that $\Delta<0$ and the second-order condition is now necessarily satisfied.

${ }^{25}$ This follows because in this case $c_{1}>c_{2}$ holds without one having to assume that $\partial \widetilde{k} / \partial T_{1} \geq 0$. The proofs of $\eta>0$ and $d_{2}>d_{1}$ are as in the unrestricted case.

${ }^{26}$ We have

$$
\frac{\partial \widetilde{k}}{\partial P_{1}}+\frac{\partial \widetilde{k}}{\partial P_{2}}=\frac{\pi^{\prime}(k)\left[v^{\prime}\left(d_{2}\right)-v^{\prime}\left(d_{1}\right)\right]}{(-\Delta)} .
$$

With $d_{2}>d_{1}$, the concavity of $v(\cdot)$ implies $v^{\prime}\left(d_{2}\right)-v^{\prime}\left(d_{1}\right)<0$, and the above expression is negative. 
they are old. We can now state, unambiguously, that as long as taxes are independent of the number of children, less resources are transferred to the future relative to the first best.

We summarize these results as

Proposition 4 Assume $T_{1}=T_{2}=T$ :

(i) The constraint implies $c_{1}>c_{2}$ thus reversing the corresponding (unconstrained) second-best finding on first-period consumption levels. The other second-best results continue to hold. That is, pension benefits increase with the number of children so that $d_{2}>d_{1} ;$ and that investment in children and the average fertility rate are less than their corresponding first-best levels.

(ii) Reducing $T_{2}$ and increasing $T_{1}$ from $T$ are welfare improving (if $\partial \widetilde{k} / \partial T_{1} \geq 0$ ).

(iv) Less resources are transferred to the future relative to the first best.

\section{Two polar cases}

Finally, it will be instructive to contrast the lessons of our model with those obtained in two polar cases: one where fertility is controlled in a deterministic way, and the other where fertility is random and purely exogenous. Consider first the case where fertility is perfectly controllable. Clearly, in a deterministic environment, there is no need to provide insurance. One only needs to worry about incentives and ensure the "correct" choice of $k$. A simple formalization of this idea within our model is to assume that $k$ takes only two values $k \in\left\{k_{1}, k_{2}\right\}$, and that $k_{1}$ leads to $n_{1}$ and $k_{2}>k_{1}$ to $n_{2}$. Assume further that the rate of return to storage is low enough that a PAYGO with $k_{1}$ is preferable to storage. Then, with children having no intrinsic benefits, parents will always choose $k_{1}$ even when $k_{2}$ happens to be optimal if contributions and pensions are independent of the number of children. However, with $n_{i}$ being observable, $k_{i}$ will also be observable. One should then be able to fully circumvent the potential moral hazard problem and ensure that the first-best outcome is attained. To align the individuals' and the society's preferences, the government should impose the ex-ante contribution/benefit package of $\left(T_{1}, P_{1}\right)$ or $\left(T_{2}, P_{2}\right)$, depending on the number of their 
number children, on all individuals. The contributions and benefits tax are set such that the optimal intertemporal allocation rule $u^{\prime}\left(y-k_{i}-T_{i}-\theta n_{i}\right)=n_{i} v^{\prime}\left(P_{i}\right)$, and the government's budget constraint $P_{i}=n_{i} T_{i}$, are satisfied. The individuals would then choose $k \in\left\{k_{1}, k_{2}\right\}$ such that $U_{i}=u\left(y-k_{i}-T_{i}-\theta n_{i}\right)+v\left(n_{i} T_{i}\right)$ is maximal; precisely as the society would want to.

Consider next the other extreme setting in which there is no control over fertility. There is no moral hazard (incentive) problem here and the optimal policy (whether $k$ is observable or not) requires $k=0$ and full insurance. This is precisely the outcome under the structure of our model when $\pi^{\prime}(k)=0$, where the optimal social security system fully insures parents against the fertility uncertainty. The government chooses its pension benefits $P$ and lump-sum tax payments $T_{1}$ and $T_{2}$ to ensure that its budget constraint is satisfied, $c_{1}=c_{2}(=c), d_{1}=d_{2}(=d)$, and $u^{\prime}(c)=\bar{n}(0) v^{\prime}(d)$. These values implement the optimum as the individuals would choose $k=0, c$ and $d$. (we are again assuming that the rate of return on storage is low enough that it is dominated by the PAYGO scheme). The first- and second-best solutions derived in this paper offer a compromise between these two extreme cases.

\section{Conclusion}

The PAYGO social security has traditionally been studied as if the rate of fertility were given or at least not controllable. More recently, a series of papers have focused on the endogeneity of fertility and the need to make parents responsible when their behavior have social externalities. In the case of PAYGO pension plans, social externalities tend to be positive implying that the laissez-faire generates a suboptimal population growth. In other cases, such as the "Tragedy of the Commons," social externalities are negative and population growth is excessive. Making people responsible for their fertility raises problems when the control is only partial because fertility involves some randomness. It is then important to insure parents against fertility shocks they are not responsible for.

We have shown that, with positive externalities, one should grant parents who have more children larger pension benefits. At the same time, the parents' contributions 
must be linked negatively to their investment in children if the investments are publicly observable, and to the number of children if investments are not observable. With observability, the outcome is characterized by full insurance with all parents enjoying identical consumption levels regardless of their number of children, when they work as well as when they are retired. In the absence of observability, families with more children should be more than compensated for the extra cost of children so that they can enjoy a higher level of first-period consumption. Moreover, the optimal level of investment in children, and the resulting average fertility rate, will be less than their corresponding first-best levels. Finally, we have shown that, except for the extra compensation result, all other second-best results carry over to situations where payroll taxes cannot depend on the number of children. 


\section{References}

[1] Abio, G., G. Mahieu and C. Patxot, (2004), On the optimality of PAYG pension systems in an endogenous fertility setting, Journal of Pension Economics and Finance, 3, 35-62.

[2] Bental, B., (1989), The old age security hypothesis and optimal population growth, Journal of Population Economics, 1, 285-301.

[3] Cigno, A., (1991), The Economics of the Family, Clarendon Press, Oxford.

[4] Cigno, A., Luporini, A. and A. Pettini, (2003), Transfers to families with children as a principal-agent problem, Journal of Public Economics, 87, 1165-1177.

[5] Cigno, A., and A. Luporini, (2003), Optimal policy with heterogeneous families, asymmetric information and stochastic fertility, paper presented at CESifo Venice Summer Institute.

[6] Cremer, H., F. Gahvari, and P. Pestieau, (2003), Stochastic fertility, moral hazard, and the design of pay-as-you-go pension plans, paper presented at CESifo Venice Summer Institute.

[7] Cremer, H., F. Gahvari, and P. Pestieau, (2004), Pensions with heterogeneous agents and endogenous fertility, mimeo.

[8] Diamond, P.A., (1965), National debt in a neoclassical growth model, American Economic Review, 55, 1125-1150.

[9] Fenge, R. and V. Meier, (2005), Pensions and fertility incentives, Canadian Journal of Economics, 38, 28-48.

[10] Kolmar, M., (1997), Intergenerational redistribution in a small open economy with endogenous fertility, Journal of Population Economics, 10, 335-356.

[11] Samuelson, P.A., (1958), An exact consumption-loan model of interest with or without the social contrivance of money, Journal of Political Economy, 66, 467482. 
[12] Sinn, H.W., (2004), The pay-as-you-go pension system as fertility insurance and an enforcement device, Journal of Public Economics, 88, 1335-1357.

[13] van Groezen, B., T. Leers and L. Meijdam, (2000), Family size, looming demographic changes and the efficiency of social security reform, CentER Working Paper no 2000-27, Tilburg.

[14] van Groezen, B., T. Leers and L. Meijdam, (2003), Social security and endogenous fertility: pensions and child allowances as Siamese twins, Journal of Public Economics, 87, 233-251. 